\title{
Development of Slewing Mirror Telescope Optical System for the UFFO-pathfinder
}

Jeong, S.; Nam, J.W.; Ahn, K.-B.; Park, I.H.; Kim, S.-W.; Lee, J.; Lim, H.; Brandt, Søren; Budtz-Jørgensen, Carl; Castro-Tirado, A.J.

Total number of authors:

30

Published in:

E A S Publications Series

Link to article, DOI:

$10.1051 /$ eas $/ 1361090$

Publication date:

2013

Link back to DTU Orbit

Citation (APA):

Jeong, S., Nam, J. W., Ahn, K-B., Park, I. H., Kim, S-W., Lee, J., Lim, H., Brandt, S., Budtz-Jørgensen, C., Castro-Tirado, A. J., Čhen, P., Cho, M. H., Choi, J. N., Grossan, B., Huang, M. A., Jung, A., Kim, J. E., Kim, M. B., Kim, Y. W., ... Yashin, I. (2013). Development of Slewing Mirror Telescope Optical System for the UFFOpathfinder. E A S Publications Series, 61, 561-565. https://doi.org/10.1051/eas/1361090

\section{General rights}

Copyright and moral rights for the publications made accessible in the public portal are retained by the authors and/or other copyright owners and it is a condition of accessing publications that users recognise and abide by the legal requirements associated with these rights.

- Users may download and print one copy of any publication from the public portal for the purpose of private study or research.

- You may not further distribute the material or use it for any profit-making activity or commercial gain

- You may freely distribute the URL identifying the publication in the public portal 


\title{
DEVELOPMENT OF SLEWING MIRROR TELESCOPE OPTICAL SYSTEM FOR THE UFFO-PATHFINDER
}

\author{
S. Jeong ${ }^{1}$, J.W. Nam ${ }^{2}$, K.-B. Ahn ${ }^{3}$, I.H. Park ${ }^{5}$, S.-W. Kim ${ }^{3,4}$, J. Lee ${ }^{5}$, \\ H. Lim ${ }^{5}$, S. Brandt ${ }^{6}$, C. Budtz-Jørgensen ${ }^{6}$, A.J. Castro-Tirado ${ }^{7}$, \\ P. Chen ${ }^{2}$, M.H. Cho ${ }^{1}$, J.N. Choi ${ }^{3}$, B. Grossan ${ }^{8}$, M.A. Huang ${ }^{9}$, A. Jung ${ }^{1}$, \\ J.E. $\operatorname{Kim}^{1}$, M.B. $\operatorname{Kim}^{5}$, Y.W. Kim ${ }^{1}$, E.V. Linder ${ }^{8,11}$, K.W. Min ${ }^{10}$, \\ G.W. Na ${ }^{1}$, M.I. Panasyuk ${ }^{12}$, J. Ripa ${ }^{5}$, V. Reglero ${ }^{13}$, G.F. Smoot ${ }^{8,11}$, \\ J.E. Suh ${ }^{1}$, S. Svertilov ${ }^{12}$, N. Vedenkin ${ }^{12}$ and I. Yashin ${ }^{12}$
}

\begin{abstract}
The Slewing Mirror Telescope (SMT) is the UV/optical telescope of UFFO-pathfinder. The SMT optical system is a RitcheyChrétien (RC) telescope of $100 \mathrm{~mm}$ diameter pointed by means of a gimbal-mounted flat mirror in front of the telescope. The RC telescope has a $17 \times 17 \operatorname{arcmin}^{2}$ in Field of View and 4.3 arcsec resolution (full width half maximum of the point spread function) The beam-steering
\end{abstract}

\footnotetext{
1 Department of Physics, Ewha Womans University, 11-1 Daehyun-dong, Seoul 120-750, Korea

2 Department of Physics, National Taiwan University, 1 Roosevelt Road, Taipei, 106, Taiwan

3 Department of Astronomy, Yonsei University, 134 Shinchon-dong, Seoul 120-749, Korea

${ }^{4}$ Yonsei University Observatory, Yonsei University, 134 Shinchon-dong, Seoul 120-749, Korea

${ }^{5}$ Department of Physics, Sungkyunkwan University, Seobu-ro, Jangangu, Suwonsi, Gyeongido 440-746, Korea

${ }^{6}$ National Space Institute Astrophysics, Technical University of Denmark, 2800 Kgs, Lyngby, Denmark

${ }^{7}$ Instituto de Astrofisica de Andalucia (IAA-CSIC), PO Box 03004, 18080 Granada, Spain

8 University of California at Berkeley, Space Sciences Laboratory, 7 Gauss Way, Berkeley, CA 94720, USA

9 Department of Energy Engineering, National United University, 1, Lienda, Miaoli, Taiwan, 36003

${ }^{10}$ Korea Advanced Institute of Science and Technology, 291 Daehak-ro, Yuseong-gu, Daejeon 305-701, Korea

11 Institute for the Early Universe, Ewha Womans University, 11-1 Daehyun-dong, Seoul 120-750, Korea

12 Skobeltsyn Institute of Nuclear Physics of Lomonosov, Moscow State University, Leninskie Gory 119234, Russia

13 Universidad de Valencia, GACE, Edif. de Centros de Investigacion, Burjassot, 46100 Valencia, Spain
} 
mirror enables the SMT to access a $35 \times 35$ degree region and point and settle within $1 \mathrm{sec}$. All mirrors were fabricated to about 0.02 wavelengths RMS in wave front error (WFE) and $84.7 \%$ average reflectivity over $200 \mathrm{~nm} \sim 650 \mathrm{~nm}$. The RC telescope was aligned to 0.05 wavelengths RMS in WFE (test wavelength $632.8 \mathrm{~nm}$ ). In this paper, the technical details of the RC telescope and slewing mirror system assembly, integration, and testing are given shortly, and performance tests of the full SMT optical system are reported.

\section{Introduction}

To measure the early photons from Gamma-Ray Bursts (GRBs), the Ultra-Fast Flash Observatory was proposed [1]. As the first step, the UFFO-pathfinder instrument has been developed and is scheduled to be launched 2013 as one of science payloads onboard the Lomonosov Russian satellite. The UFFO-pathfinder (Park et al. 2012) consists of two instruments, the UFFO Burst Alert and Trigger Telescope (UBAT) (Park et al. 2012; Jung et al. 2011; Nim et al. 2011; Na et al. 2011) for X-ray trigger and localization and Slewing Mirror Telescope (SMT; Jeong et al. 2013). When UBAT provides a trigger signal and approximate coordinates of a GRB to SMT, the SMT slewing mirror rotates quickly to the target coordinate and brings images of the UV/optical counter parts of GRB into the instrument FOV (Field of view) faster than the current GRB instruments in space and ground. The SMT optical system in UFFO-pathfinder is the first proof to realize such a measurement concept in GRB observation. In this paper, especially the technical details of SMT optical system, i.e. slewing mirror stage and RC telescope are presented shortly.

\section{SMT RC telescope and slewing mirror assembly}

The SMT optical system requirements are summarized in Table 1. The RitcheyChrétien (RC) telescope has a primary mirror (M1) of $100 \mathrm{~mm}$ in diameter and -1.01 in conic coefficient and a secondary mirror (M2) of $20 \mathrm{~mm}$ in diameter and -1.83 in conic coefficient. The telescope has $1.14 \mathrm{~m}$ effective focal length and fits within $200 \mathrm{~mm}$ SMT length budget in UFFO-pathfinder. It produces $17 \times$ $17 \operatorname{arcmin}^{2}$ in instantaneous FOV (IFOV) on the detector and almost diffraction limited performance, with a Modulation Transfer Function (MTF) of 0.77 at $22.52 \mathrm{~mm}^{-1}$ Nyquist frequency and an on-axis RMS spot radius of $2.48 \mu \mathrm{m}$. Its spot diagrams over the SMT FOV are well within the Airy disk of $400 \mathrm{~nm}$ in diameter. The optics design satisfies all the $\mathrm{RC}$ telescope performance requirements as listed in Table 1. To minimize the stress on the M1 caused by a mismatch in thermal expansion coefficient (CTE) between the Invar flexure and the aluminum support structure, the M1 is mounted to the M1 support plate via 3 bi-pod flexures with $12 \mathrm{~mm}$ long, $2 \mathrm{~mm}$ wide, and $1.5 \mathrm{~mm}$ thick. The M2 cell is then mounted to the M1 support plate by 4 spider arms. The M2 cell has an invar spider core with 3 flexure blades. The final resulting RMS system WFE of the RC telescopes was found to be $22.3 \mathrm{~nm}$ for $633 \mathrm{~nm}$ in wavelength that is well within WFE requirement of $<0.15$ waves. 
Table 1. Requirements for SMT optics.

\begin{tabular}{cc}
\hline SMT optics & Slewing mirror and RC telescope \\
\hline Aperture size & $10 \mathrm{~cm}$ \\
Slewing coverage & $70 \times 70 \operatorname{arcdeg}^{2}$ \\
Slewing speed & $>30 \mathrm{deg} / \mathrm{sec}^{2}$ \\
Detector FOV & $17 \times 17 \operatorname{arcmin}^{2}$ \\
Angular resolution & 4 arcsec \\
Advanced resolution & $0.5 \operatorname{arcsec}($ after centroiding $)$ \\
Modulation Transfer Function & $>0.4$ at $22.52 \mathrm{~mm}^{1}$ \\
Sensitive wavelength & $200-650 \mathrm{~nm}$ \\
Mass & $3 \mathrm{~kg}$ \\
Volume & $600(\mathrm{l}) \times 320(\mathrm{w}) \times 200(\mathrm{~h}) \mathrm{mm}^{3}$ \\
\hline
\end{tabular}

The slewing mirror stage has an aluminum support ring and RTV566 pads are inserted between the ring and mirror around its peripheral, front and back surfaces. A two axes gimbal stage is constructed with stepper motors and rotary encoders. Using the harmonic drive gear, its step size is controlled with $\sim 4$ arcsec precision and the rotation angle can be controlled precisely to 2 arcmin in accuracy. The 2 axis stage can be rotated from $+22.25^{\circ}$ to $-21.47^{\circ}$ in inner axis and from $+66.26^{\circ}$ to $-2.25^{\circ}$ in outer axis on average. It allows for the full $\pm 35^{\circ}$ in sky coverage. The total mass of slewing mirror assembly is measured to about $1.85 \mathrm{~kg}$, satisfying the mass requirement of $<2 \mathrm{~kg}$. All mirrors are made of Zerodur substrates, coated with $\mathrm{SiO}_{2}+\mathrm{Al}$ for a reflexivity $\mathrm{R}_{\text {avg }}>85 \%$. The slewing mirror Zerodur blank is light weighted $482 \mathrm{~g}$ to satisfy the mass budget $<0.6 \mathrm{~kg}$. The developed SMT optical system is presented in Figure 1.

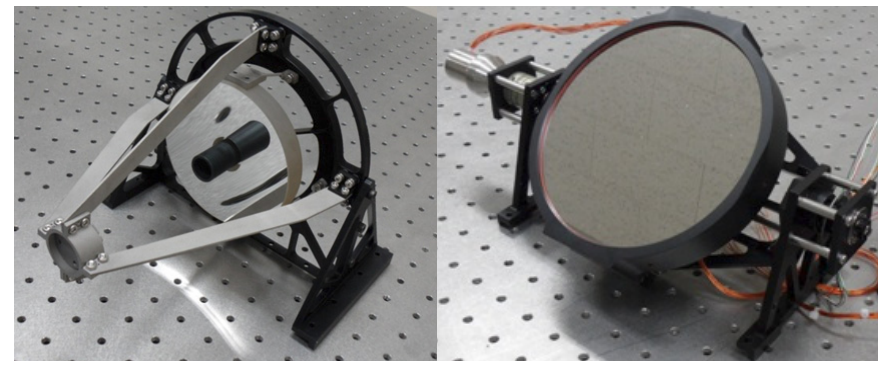

Fig. 1. Manufactured SMT optics system, RC telescope (left) and slewing mirror stage (right).

\section{System validation test of SMT optics}

The flight model SMT performance was validated in Russia. The pre-flight model of RC telescope was used as a collimator. A He-Ne laser beam with $635 \mathrm{~nm}$ 
was focused on the PFM RC telescope focus point. It was then diverged before being collimated to a parallel beam to feed the slewing mirror that redirected it to the M1. The beam was subsequently reflected by M2, and then focused onto the focal plane. The resulting focal images of the $4.3 \operatorname{arcsec}(Y$-slice, Gaussian fit $1 \sigma$ ) PSF is presented in Figure 2.

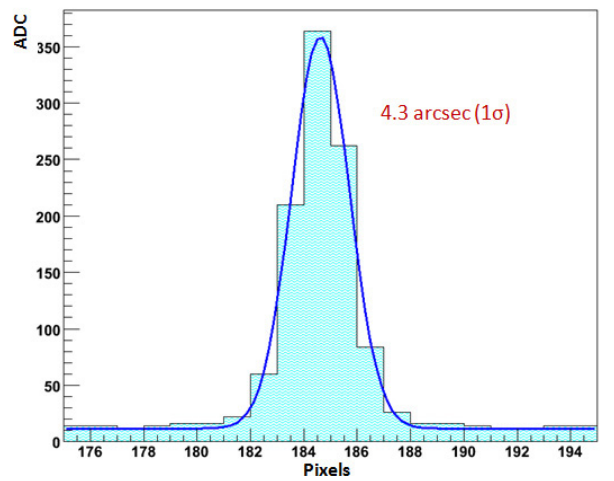

Fig. 2. Point spread function of flight model SMT [2].

\section{Conclusion}

The UFFO project has proposed for the first time the systematic exploration of UV/optical GRB light curves far earlier than 60 seconds after the localization of GRBs by X-ray observations. It is based on a novel concept of Slewing Mirror Telescope (SMT) that utilizes a fast slewing or tracking mirror, instead of controlling the attitude of a whole satellite or telescope body, to point and track the target GRB. It has $10 \mathrm{~cm}$ aperture and provides a $\sim 4.3 \operatorname{arcsec}(1 \sigma$ width $)$ PSF over $17 \times 17 \operatorname{arcmin}^{2}$ in IFOV. And the angular resolution will be recovered up to 0.5 arcsec after the centroiding algorithm. This IFOV can be expanded to cover \pm 35 degrees in operational FOV within about $1 \mathrm{~s}$ after the trigger using slewing mirror. The SMT optical system has been designed, constructed at first time and its SMT flight instrument is now integrated in the Lomonosov satellite pending launch in 2013.

This research was supported by the Korean Creative Research Initiatives (RCMST) of MEST/ NRF, the Basic Science Research program of MEST/NRF (2010-0025056), the World Class University program of MEST/NRF (R32-2009-000-10130-0), the Spanish MINECO project AYA2009-14027-C05-01, AYA2011-29936-C05-01, AYA-2012-39727-C03-01 and AYA 2009-14000-C0301/ESP, Taiwan's National Science Council Vanguard Program (100-2119-M-002-025) LeCosPA of National Taiwan University, Program of development of Lomonosov Moscow State University and Korean programs NRF 2012-0006632, 2010-0029390 and Yonsei-KASI joint research for the Frontiers of Astronomy and Space Science Program 2012. We thank Samsung Electronics (Super-precision Optics Lab., Imaging Division) for production of SMT mirrors, the National Space Organization (NSPO) of Taiwan and Nauchno-issledovatelskij institut elektromehaniki 
(NIIEM) of Russia for instrument space qualification, SCHOTT KOREA for consulting on the design of a light-weight mirror, and Dr. Yang of KRISS and H. K. Lee of Samsung for valuable discussions.

\section{References}

Jeong, S., et al., 2013, Opt. Express, accepted

Jung, A., Ahmad, S., Ahn, K.-B., et al., 2011 [arXiv:1106.3802]

Kim, J.E., Lim, H., Jung, A., et al., 2011 [arXiv:1106.3803]

Na, G.W., Ahn, K.-B., Choi, H.S., et al., 2011 [arXiv:1106.3804]

Park, I.H., et al., 2012, submitted to New J. Phys. 